\title{
A Self-Adapting Healthcare Information Infrastructure Using Mobile Computing Devices
}

James K. Archibald

james_archibald@byu.edu

Eric S. Hall

enrique.hall@gmail.com

Charles D. Knutson

knutson@cs.byu.edu

David K. Vawdrey

Follow this and additional works at: https://scholarsarchive.byu.edu/facpub

Part of the Computer Sciences Commons

\section{Original Publication Citation}

David K. Vawdrey, Eric S. Hall, and Charles D. Knutson. "A Self-Adapting Healthcare Information Infrastructure Using Mobile Computing Devices." Proceedings of the 5th IEEE International Workshop in Enterprise Networking and Computing in Healthcare Industry (HealthCom 23), Santa Monica, California, June 6-7, 23.

\section{BYU ScholarsArchive Citation}

Archibald, James K.; Hall, Eric S.; Knutson, Charles D.; and Vawdrey, David K., "A Self-Adapting Healthcare Information Infrastructure Using Mobile Computing Devices" (2003). Faculty Publications. 492.

https://scholarsarchive.byu.edu/facpub/492 


\title{
A Self-Adapting Healthcare Information Infrastructure Using Mobile Computing Devices
}

\author{
David K. Vawdrey*, Eric S. Hall*, Charles D. Knutson*, and James K Archibald ${ }^{\dagger}$ \\ *Department of Computer Science \\ tDepartment of Electrical and Computer Engineering \\ Brigham Young University, Provo, Utah 84602 \\ Email: dkv@email.byu.edu
}

\begin{abstract}
Despite recent improvements in the gathering and sharing of patient medical information among healthcare providers, there remains a gap in the electronic medical record infrastructure. Patient data is not available in some situations, either because the infrastructure is inaccessible (as in a natural disaster) or because there is no way to link the patient to the infrastructure (e.g., the patient cannot supply necessary identification information). This paper describes the Poket Doktor System, an architecture that allows an individual to carry personal electronic medical information on a wireless handheld device such as a smart card, cell phone, or PDA. Medical workers can obtain this information wirelessly using handheld devices, desktop computers, network access points, etc. In this way, patients play an active role in the medical information infrastructure, resulting in a better healthcare delivery system.
\end{abstract}

\section{INTRODUCTION}

The highly-publicized 1999 Institute of Medicine report, To Err Is Human: Building a Safer Health System, suggests that between 44,000 and 98,000 Americans die each year as a result of medical errors [1]. The report states that deaths due to medical errors in 1998 exceeded the number attributable to motor vehicle accidents $(43,458)$, breast cancer $(42,297)$, and AIDS $(16,516)$. Total national costs associated with medical errors resulting in injury are estimated to be between $\$ 17$ billion and $\$ 29$ billion annually.

The predominant cause of medical errors in most cases is not substandard training or gross negligence on the part of healthcare professionals. Errors with negative patient outcomes are usually the result of complex combinations of actions by care providers-including the following of standard protocols as well as both minor and major oversights and indiscretions. A common thread in almost all documented medical mistakes is the lack of detailed and timely information about the patient [2] [3].

When a patient is treated by a healthcare provider, specific information exists that would facilitate effective and appropriate treatment if it were available. Such information includes patient medical history and existing conditions, results of medical tests and examinations, allergies, and current prescriptions. Without access to accurate and detailed patient record information, medical professionals cannot provide effective care [4].

This paper discusses the Poket Doktor System, which uses personal electronic devices to store and wirelessly communi- cate a patient's digital medical record to healthcare providers. The architecture of the system includes the following components:

- Electronic Patient Devices (EPDs)-handheld devices carried by patients that contain personal medical information databases;

- Healthcare Provider Devices (HPDs), which may be handheld computers, desktop workstations, or hospital network access points; and

- Quality of Transport (QoT), a communications link management technology that allows EPDs to exchange information with HPDs using multiple transport protocols operating over various transmission media.

The remainder of this paper is structured as follows. Section II provides background information and outlines related work. Section III describes the current state of the electronic medical record communication infrastructure and identifies key weaknesses in the current model. Section IV introduces the notion of patient participation in the infrastructure using handheld electronic devices. Section V explains the Poket Doktor System architecture in detail. Section VI presents a proof-of-concept prototype implementation of the system, and Section VII offers conclusions.

\section{BACKGROUND}

Millions of people suffer from medical conditions that should be made known to healthcare practitioners prior to treatment. Paramedics and emergency room doctors cannot provide optimal care without sufficient knowledge of a patient's medical history. Lacking vital patient information, medical professionals are forced either to delay treatment or rely on faulty or insufficient data. Unfortunately, timely access to this information is often impossible, especially in emergency situations.

Traditionally, patient medical records-including medical histories, results of medical tests and examinations, and emergency contact information-are maintained in paper files stored at the offices of individual healthcare providers [5]. Sharing has been burdensome, typically requiring the physical delivery of duplicate materials.

With the growth of electronic medical record (EMR) systems over the past four decades, it is becoming easier to communicate and synchronize medical record information 
between the disparate entities involved in the treatment of patients [6] [7] [8]. Recent efforts have focused on providing connectivity among provider institutions, record repositories, and practitioners [9]. Improvements in communication technologies (both wired and wireless) offer improved methods for transmitting EMR information, while standards developed within the medical informatics community (such as HL7 [10], DICOM [11], LOINC [12], and SNOMED [13]) provide consistency in formatting and storing data.

The 2001 Report, A Strategy.for Building the National Health Information Infrastructure published by the U.S. Department of Health and Human Services suggests that the United States urgently needs a comprehensive National Health Information Infrastructure and that "ready access to relevant, reliable information would greatly improve everyone's ability to address personal and community health concerns" [14]. The report further states that such a system is now technologically feasible, and its creation will have a marked impact on the effectiveness, efficiency, and quality of healthcare.

Several researchers are involved in specific projects focused on enhancing and expanding methods for storing and exchanging EMR data. The Ward-in-Hand project is improving the communication of records within a hospital environment by supplying medical practitioners with mobile computers [15]. These wireless-enabled devices give doctors and nurses immediate access to patient records stored on a centralized server.

A recent study conducted by IBM Healthcare Consulting and the University of Michigan Hospitals and Health Centers examined the benefits of using handheld computers to bridge the gap between on-call practitioners and hospital record databases [16]. The study concluded that a flexible infrastructure is required, and there is no "one size fits all" solution for a given situation.

Other researchers have examined the potential of extending the EMR infrastructure to allow access to hospital resources from ambulances equipped with cellular and other wireless communication technologies [17] [18] [19].

\section{The ElECtronic Medical INFORMATION INFRASTRUCTURE (EMII)}

As a result of these on-going efforts, electronic medical information now has the potential to be shared among healthcare practitioners in hospitals, doctor's offices, pharmacies and other facilities. Figure 1 illustrates what we have termed an Electronic Medical Information Infrastructure (EMII). Broadreaching EMIIs are beginning to allow patient EMR data to be freely and quickly exchanged among multiple healthcare providers.

The increased information flow among medical facilities enables the healthcare delivery system to become patientcentric, with accurate patient EMR data immediately available at the point of care [20]. In seeking after this vision, however, researchers have largely ignored a key issue: connecting the patient to the infrastructure. Despite significant progress in sharing information via wired and wireless EMIIs, it may be

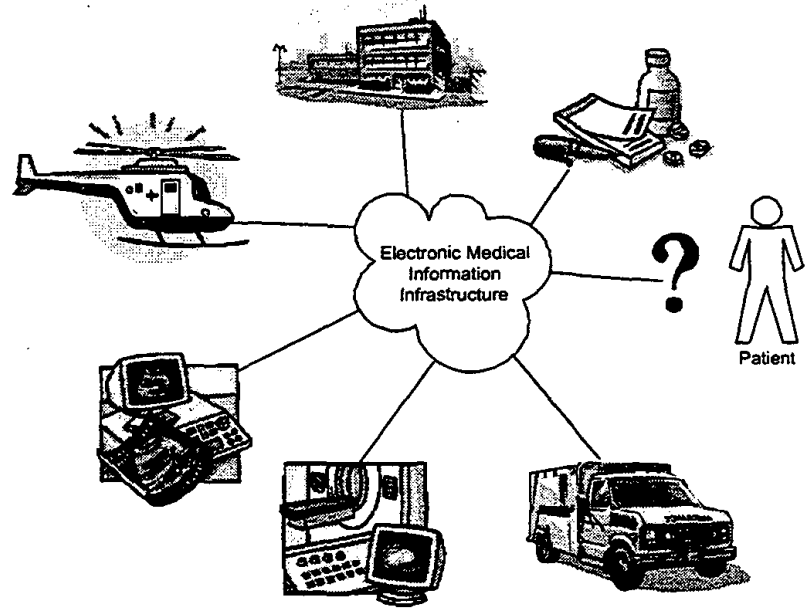

Figure 1. Connectivity within the emerging Electronic Medical Information Infrastructure (EMII).

impossible for emergency workers to access an EMII if they are out of range or the infrastructure is damaged. In another scenario, if a patient is unconscious or incoherent, medical professionals may not be able to properly identify her in order to obtain her medical record via the infrastructure.

To overcome these problems, some groups are examining systems that keep personal EMR data in the hands of the patient [21] [22]. In this patient-centered model, medical practitioners can access critical patient information even if they cannot connect with or identify the patient to the EMII. One example of this approach is the computerized Personal Information Carrier (PIC) being developed by the U.S. Armed Forces [23]. Maintaining the same form factor as traditional military dog tags, the PIC can store a soldier's identification information and up to $8 \mathrm{MB}$ of audio, video, and text-based EMR data.

While the PIC concept is well-suited for military applications, expanding this idea to civilian markets raises serious concerns in locating and identifying patient devices, maintaining privacy and confidentiality, and establishing standards to guarantee interoperability [24]. For example, when treating an unconscious patient, medical workers typically do not have the option of searching the patient's personal belongings to locate a device containing EMR data. In short, when a patient cannot come to the infrastructure (e.g., the patient is unable to communicate the relevant personal information necessary to access appropriate medical records), or the infrastructure is unavailable (as in a natural disaster), the quality of healthcare for that patient may suffer.

\section{The Patient's Role in the EMII}

Because individuals are mobile and EMIIs typically are not, there exists a gap between patient and infrastructure. Solving this problem requires a change in thinking from current models. For the healthcare delivery system to become truly patient- 


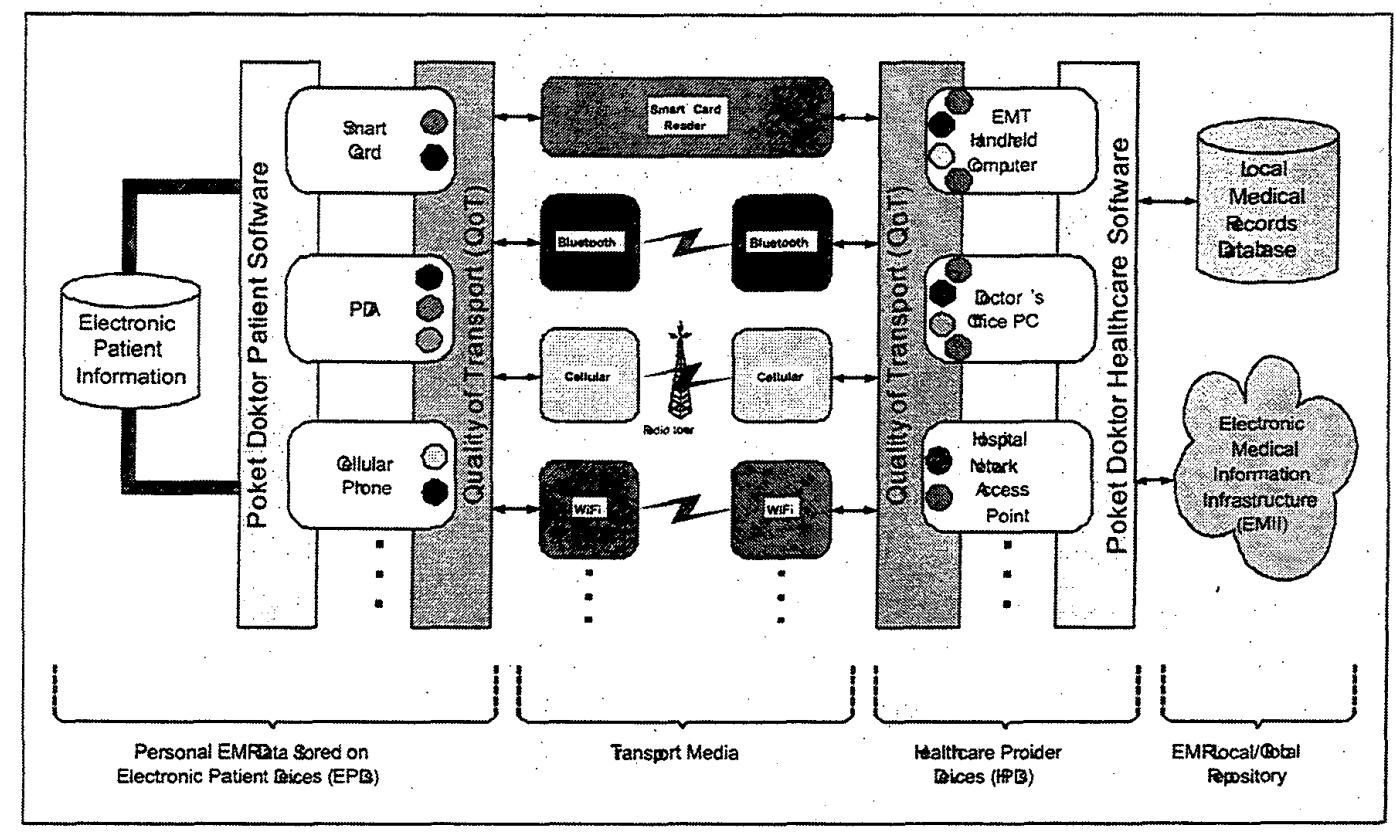

Figure 2. Poket Doktor System Architecture.

centric, patients themselves must participate as an active and dynamic source of information within the infrastructure. To achieve this aim, we propose a system wherein healthcare workers are able to access critical information carried by patients via personal electronic devices. Two advances in technology make this possible:

1) Proliferation of mobile computing devices. Mobile computing devices (such as smart cards, cellular phones, PDAs, digital wristwatches and pagers) have become smaller, more powerful, and more capable of storing information [25]. As a consequence, the use of mobile computing devices has exploded in the past 10 years. With the increasing number of mobile computing platforms, some have argued that these will eventually be distilled into a single multi-purpose device. Some merging of platforms will certainly take place, but specialized devices likely will continue to thrive. For example, modern cellular phones often include features traditionally found in PDAs-a clear example of technologies merging. However, a hybrid cell phone/PDA device does not eliminate the market need for wristwatches or pagers.

2) Ubiquitous wireless data communication technologies. Wireless transport protocols such as Bluetooth, WiFi (IEEE 802.11), UltraWideband, and cellular communication are becoming increasingly widespread, and emerging standards are well-suited for use in mobile devices. Each of these transports is designed to meet the needs of specific usage models, so it is unlikely that a single technology will become predominant. Unless the world of wireless data communication converges to a single application environment, dealing with multiple transports will remain an important issue. [26].

Along with the challenges associated with multiple platforms and communication technologies comes a unique opportunity to connect individual patients with the EMII.

To address these challenges requires a broad, flexible architecture that allows a patient's personal electronic devices to store and wirelessly communicate EMR data to medical professionals. The Poket Doktor System provides such a framework, enabling individuals to quickly and securely transfer personal medical information to healthcare workers. The system solves many of the problems associated with the current state of the medical information infrastructure by operating either in conjunction with or independently of existing EMIIs.

\section{ARCHITECTURE OF A PATIENT-CENTRIC INFRASTRUCTURE}

The Poket Doktor System architecture is designed to allow an individual to carry personal electronic medical information on a wireless handheld device such as a cell phone, PDA, or enhanced smart card. Medical workers can obtain this information wirelessly using handheld devices, desktop computers, network access points, etc. In this way, patients become an active component of the medical information infrastructure.

Figure 2 diagrams the functional components and communications model for the Poket Doktor System architecture. Electronic Patient Devices (EPDs) are small, portable devices 
used to store and communicate personal EMR data. They may be based on a number of handheld computing platforms including smart cards, PDAs, or cellular telephones. EPDs include a database for electronically storing a patient's medical information, system and application software, and one or more wireless transport mechanisms for exchanging data.

Healthcare Provider Devices (HPDs) are computing platforms used by medical practitioners. Such platforms may include PDAs, desktop or laptop personal computers, and hospital network access points. Application software on HPDs allows healthcare providers to exchange data wirelessly with EPDs. In addition, HPDs are able to access and synchronize data with EMR repositories both locally and via external infrastructures.

Communication between EPDs and HPDs is managed by a technology we call Quality of Transport (QoT) [27]. QoT permits a device to dynamically and transparently change underlying data transport protocols without interrupting the communication session between applications. Using QoT provides a mechanism for maintaining communication under harsh environmental conditions and for optimizing communication based on desired specifications.

These key components of the Poket Doktor System are further described in following subsections.

\section{A. Electronic Patient Devices (EPDs)}

The Poket Doktor Architecture specifies that an EPD in- clude a database for storing a patient's critical medical information, system and application software, and one or more wireless transport media. These components are described below.

\section{- Patient Information Database (PID)}

The Patient Information Database is a repository of personal EMR data that scales to the capacity of the host EPD. Sufficient memory and computing power allow the patient to store a large, comprehensive medical record. With space and processing limitations the PID may be less complete, containing only the most vital information necessary for emergency treatment.

\section{- Patient Application Software}

The application software resides on the EPD and manages the medical information contained in the database, updating it when needed and supplying it electronically when requested by an authorized HPD.

- Transceivers and transport protocols

At least one wireless transceiver must be available for use by the patient application software. Supported transceivers range from low-power, low-cost technologies such as Bluetooth to more powerful WiFi or cellular modules. For EPDs that support multiple transceivers, QoT makes dynamic decisions concerning the best or most appropriate transport to use for a given communication session (see Section V-C).

\section{B. Healthcare Provider Devices (HPDs)}

Healthcare providers use HPDs to communicate wirelessly with patient-carried EPDs, and also to access local or external record repositories. HPDs have the following common features:

\section{- Healthcare Application Software}

The HPD's application software controls communication with patient devices. It provides a user interface and implements data formatting and parsing algorithms as well as high-level security protocols. The application software also manages the flow of information to and from local databases and accessible EMIIs.

- Transceivers and transport protocols

The HPD must support multiple transceivers for communicating with EPDs.- Since the HPD may encounter EPDs with varying communication capabilities, it must be equipped with a broad range of transceivers and transport protocols. QoT is used in the HPD to assist in establishing and managing wireless transport links, and to preserve and optimize communication sessions (see Section V-C).

\section{- Medical Information Databases}

While the EPD maintains its own minimal PID, the HPD must communicate with a local database (such as might be found in a doctor's office) or to a larger repository of information via an EMII. The local database will be typical of situations in which a doctor's office (or other healthcare facility) has its own EMR system, but is not necessarily able to access a broader infrastructure of information shared between facilities. Accessing the information infrastructure introduces a two-fold challenge: 1) managing the data connection to multiple destinations, and 2) exchanging information in a meaningful way with disparate records systems.

\section{Quality of Transport (QoT)}

The purpose of QoT is to manage underlying data connections over multiple transports in order to enhance connectivity and optimize user experience [27]. QoT is a key component in the Poket Doktor System because it increases the likelihood that exchanging information between an EPD and an HPD is successful and efficient.

The highest link management priority for QoT is to establish and preserve a connection by dynamically switching among transports when necessary. When a connection fails (or threatens to fail) QoT automatically seeks an alternate shared transport to maintain the communication session. This process is referred to as a connection downgrade. A connection upgrade occurs when a connection is already in place, but a more desirable transport becomes available. In this situation the system switches transports in order to provide the maximum quality for that connection based on some pre-defined criteria [27].

Two devices wishing to communicate must share a common session protocol in order to establish a connection. For example, in an Internet context, a web browser communicates with a web server using the shared HTTP session protocol. Session 


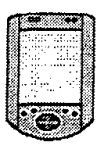

IIEVE 1

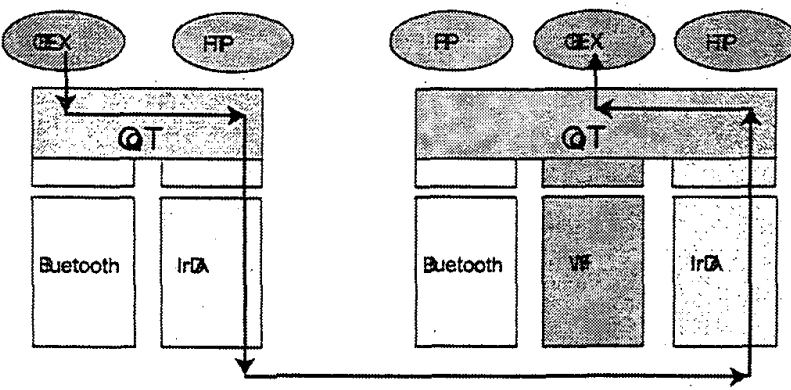

Figure 3. QoT-enabled data communication. protocols, in turn, rely on an underlying transport protocol. HTTP, for example, relies on the TCP/IP standard.

Figure 3 depicts the behavior of a QoT-enabled communication session. The two devices in this figure support HTTP and OBEX session protocols. (Device 2 also supports the FTP session protocol, although Device 1 does not.) The connection in the figure has been established using the object exchange protocol OBEX. Both devices have two underlying transports in common-Bluetooth and IrDA (infrared). IrDA is currently serving as the transport medium for the communication session between the two devices. If Bluetooth becomes more desirable for this exchange (e.g., the devices do not maintain the lineof-sight link required by IrDA), QoT will dynamically switch the transport.

As another example, consider a patient with a PDA-based EPD communicating with a Poket Doktor network access point in a hospital. Because of battery limitations, the EPD defaults to a low-power Bluetooth communication link. If the patient wanders outside the ten meter range of the hospital's Bluetooth access point, QOT automatically switches to a longer-range WiFi connection, drawing more battery power but preserving the connection.

For computing platforms that offer multiple communications transports, QoT determines the most appropriate medium to use for a given information exchange scenario. This significantly increases the likelihood of successful data transmission. In time-critical situations such as medical emergencies, QoT works transparently to preserve and optimize wireless communication channels, removing this burden from medical workers. In addition to the obvious application of QoT to the communication link between the EPD and the HPD, we expect that QoT will also have practical application to communication between healthcare devices and outside medical information infrastructures.

\section{PROOF-OF-CONCEPT IMPLEMENTATION}

The following subsections describe the first phase of Poket Doktor System implementation, consisting of the design and prototyping of a smart card-based Electronic Patient Device (EPD) and the development of application software for a simple handheld computer Healthcare Provider Device (HPD).

\section{A. Smart Card EPD}

The smart card EPD is equipped with solid-state memory and short-range wireless communication capabilities, allowing it to store and transmit EMR data. Figure 4 shows the block diagram for the Poket Doktor Smart Card EPD platform. It consists of a microcontroller-based smart card with a flat Lithium-Manganese Dioxide battery and a wireless communications module. This design provides adequate processing power, storage capacity, and communication capabilities to fit the needs of the Poket Doktor System.

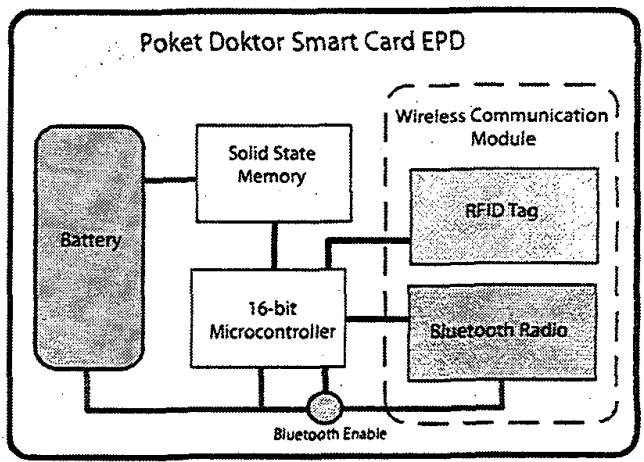

Figure 4. Poket Doktor Smart Card. block diagram.

The smart card's wireless communication subsystem consists of a Bluetooth transceiver and a Radio Frequency Identification (RFID) tag. Bluetooth wireless technology offers several features that make it a desirable medium for transmitting EMR data stored on a smart card. An emerging standard in wireless data communications, Bluetooth provides robust, medium-bandwidth communication within a 10-100 meter range. Low cost, minimal power consumption, and a small physical footprint make Bluetooth well-suited for integration into embedded devices.

Limitations associated with Bluetooth are a lengthy discovery process and excessive power consumption when communication is not desired. To alleviate these problems, we have developed a process called Rendez-Blue [28], which leverages the strengths of RFID technology to speed up the Bluetooth connection establishment process and minimize unnecessary power consumption. RFID is an automatic data capture system that uses electromagnetic or inductive coupling to transmit a small amount of data from a uniquely programmed transponder, called an RF tag, to a transceiver or reader. The RF tag may be passive, meaning that it requires no power source to transmit data (operating power is supplied by the reader). Passive RF tags provide a virtually unlimited operational 
lifetime, making them very attractive for use in the Poket Doktor Smart Card EPD.

In Rendez-Blue, RFID is used to send a "wake-up" cue to the EPD's Bluetooth radio when communication is desired (see Figure 5). At all other times, the radio is in a shutdown state, consuming no power. Using Rendez-Blue ensures that the Bluetooth module on the Poket Doktor Smart Card will respond to an inquiry and establish a connection only when it is communicating with an HPD. Thus battery life is not wasted in responding to discovery inquiries from other Bluetoothenabled devices. In addition, the wake-up cue in Rendez-Blue allows medical professionals to effectively vary the search radius, so they can discover and connect with smart card EPDs at distances ranging from a few inches to several meters. This is especially valuable for emergencies that might occur in a crowded area where many people are carrying Poket Doktor Smart Card EPDs.

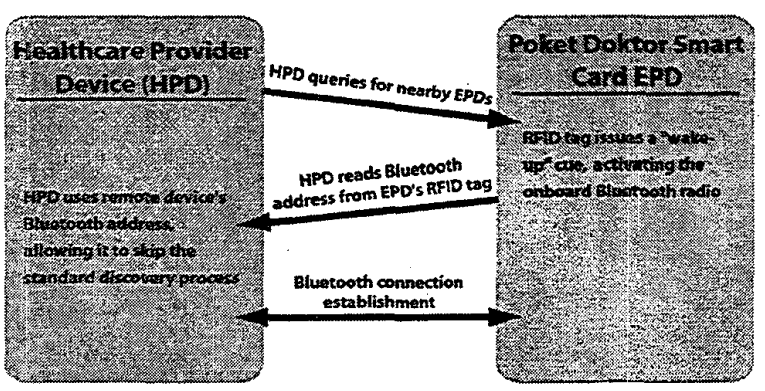

Figure 5. Rendez-Blue connection establishment procedure.

In the Rendez-Blue process, the EPD's RF tag also communicates the Bluetooth radio's hardware address to the querying device (the HPD containing the RFID transceiver). Receiving the Bluetooth address via RFID allows the HPD to bypass the standard Bluetooth inquiry process and proceed directly to connection establishment as illustrated in Figure 5. Empirical testing has shown that this aspect of the Rendez-Blue process results in a four-to-six-fold reduction in device discovery time [28].

\section{B. HPD Handheld Computer and Application Software}

Prototype development of application software has been completed, allowing desktop and handheld computers to access EMR data on Poket Doktor Smart Cards. A variety of computing platforms are currently being used in the healthcare industry to access EMR information. The Poket Doktor System is designed to ensure compatibility with existing hardware and software systems. The flexible structure of Poket Doktor application software allows straightforward integration with existing desktop terminals at hospitals and doctor's offices. In mobile devices, pervasive wireless technologies such as Bluetooth will allow HPDs to communicate with EPDs regardless of hardware platform and configuration dependencies.

The prototype HPD platform is based on the HP Jornada 720 augmented with Bluetooth and RFID communications

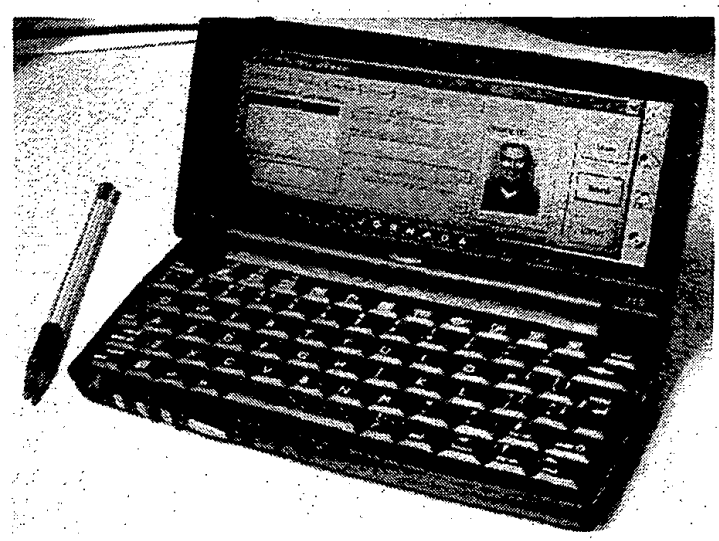

Figure 6. Handheld computer HPD based on the HP Jomada 720.

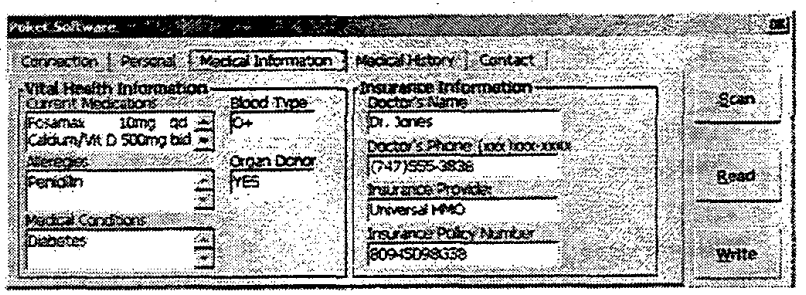

Figure 7. Screen capture of Poket Doktor software running on handheld computer.

capabilities (see Figure 6). This type of device is suitable for use at the point of care, be it an accident scene or a patient's bedside. It provides a color touch-screen display, a full keyboard, and extensive L/O capabilities.

Prototype application software is written in Embedded Visual $\mathrm{C}++$ and is compatible with a host of devices running the PocketPC operating system. The following subsections discuss user interface, data formatting, and security design decisions in development of the Poket Doktor application software.

1) Graphical User Interface (GUI): The application software's GUI allows users to issue instructions and view information in a familiar windowed environment. On-screen buttons allow a medical worker to perform functions such as discovering patient EPDs and retrieving EMR data. Figure 7 shows the user interface of the application software running on a handheld computer and illustrates the result of reading data from a patient's EPD. EMR data are grouped into general categories and each screen is separated into modifiable fields. This format provides quick access to information and a simple mechanism for updating data.

2) Data Formatting: To allow standardization and efficiency in retrieving and parsing data, the Poket Doktor application stores medical records using the eXtensible Markup Language (XML). XML is used within HL7 and other medical information standards groups to store and manage clinical information. Using XML in the Poket Doktor application takes 
advantage of existing standards and provides consistency and structure in data exchange.

3) Security: The sensitive nature of medical record information is underscored by the legal obligations of healthcare providers outlined in the 1996 HIPAA legislation [29]. Patients have a high expectation of confidentiality and an inherent distrust of storing and communicating EMR information electronically [24]. To alleviate these fears, the Poket Doktor System supports several security features to safeguard against the unauthorized access of personal data. To protect against eavesdropping, the software architecture augments standard wireless security protocols by providing secure key exchange methods and strong conventional encryption algorithms such as 3DES and AES. This allows sensitive medical information to be stored and communicated securely, preventing unauthorized devices from accessing confidential information.

In a medical emergency situation where a patient is unresponsive, it is important that authorized medical workers are able to obtain information critical to administering proper treatment. At the same time, patients may not want to allow unlimited access to personal data without consent. Poket Doktor EPDs allow the owner to selectively restrict access to certain data such as insurance information or social security number. When a card is initialized at a hospital or doctor's office, the patient may choose a password and specify certain data fields as accessible and others as restricted. The Poket Doktor application software does not display restricted fields until the card owner supplies the correct password. This selected blocking of information enables personalized medical care without sacrificing patient privacy.

\section{CONCLUSION}

The Poket Doktor System architecture provides a framework for storing and communicating Electronic Medical Record information. Patients carrying personal EMR data on handheld electronic devices can supply complete, accurate information to medical professionals using next-generation wireless technologies. Even if the patient is unconscious or unable to communicate, emergency workers can obtain critical health information to assist in diagnosis and treatment.

Storing EMR data on handheld devices improves upon traditional methods of information sharing and enables a truly patient-centric model of healthcare delivery. Along with current and future research in data security, data formatting, and embedded wireless communication devices, the Poket Doktor System will improve the quality of medical care by providing personalized health information when and where it is needed the most.

\section{REFERENCES}

[1] Institute of Medicine, To err is human: Building a better health care system. Washington, DC: National Academy Press, 2000.

[2] L. L. Leape, "Error in medicine," Journal of the American Medical Association, vol. 272, no. 23, pp. 1851-1857, 1994.

[3] T. S. Lesar, L. Briceland, and D. S. Stein, "Factors related to errors in medication prescribing," Joumal of the American Medical Association, vol. 277, no. 4, pp. 312-317, Jan. 1997.
[4] Institute of Medicine, Crossing the qualitv chasm: A new health svstem for the 21st century. Washington, DC: National Academy Press. 2001.

[5] C. Safran, D. Z. Sands, and D. M. Rind, "Online medical records: a decade of experience," Methods of Information in Medicine, vol. 38, no. 4/5, pp. 308-312, 1999.

[6] R. S. Dick, E. B. Steen, and D. E. Detmer, The computer-based patient record: an essential technologv for health care. Washington, DC: National Academy Press, 1997.

[7] J. van der Lei, "Closing the loop between clinical practice, research, and education: The potential of electronic patient records," Methods of Information in Medicine, vol. 41, no. 1, pp. 51-54, 2002.

[8] G. O. Barnett and H. J. Sukenik, "Hospital information systems," in Future Goals of Engineering in Biology and Medicine, J. F. Dickson and J. H. U. Brown, Eds. New York: Academic Press, 1969.

[9] I. S. Kohane, F. J. van Winderde, J. C. Fackler, C. Cimino, P. Kilbridge, and S. Murphy, "Sharing electronic medical records across multiple heterogeneous and competing institutions," in Proceedings of the American Medical Informatics Association Annual Fall Symposium, J. J. Cimino, Ed., 1996, pp. 608-612.

[10] "Health Level Seven." [Online]. Available: http://www.hl7.org

[11] "Digital Imaging and Communications in Medicine (DICOM)." [Online]. Available: http://medical.nema.org/dicom/2001.html

[12] "Logical Observation Identifier Names and Codes (LOINC)." [Online]. Available: http://www.regenstrief.org/loinc/

[13] "The Systematized NOmenclature of MEDicine (SNOMED)." [Online]. Available: http://www.snomed.org

[14] "Information for health: A strategy for building the national health information infrastructure," National Committee on Vital and Health Statistics, Tech. Rep., Dec. 2001.

[15] M. Ancona, E. Coscia, G. Dodero, M. Earney, V. Gianuzzi, F. Minuto and S. Virtuoso, "Ward-In-Hand: Wireless access to clinical records for mobile healthcare professionals," in TEHRE $2001 \mathrm{~m}$-Health Conference. First Annual Conference on Mobile \& Wireless Healthcare Applications, London, UK, Nov. 2001.

[16] K. C. Kimmel and A. Perry, "Environmental assessment: Wireless in healthcare," in First International Wireless Applications and Technologies Conference, Las Vegas, NV, Oct. 2001.

[17] S. Morton and O. Bukhres, "Utilizing mobile computing in the Wishard Memorial Hospital ambulatory service," in $A C M$ Symposium on Applied Computing, San Jose, CA, Feb. 1997, pp. 287-294.

[18] R. H. Katz and E. A. Brewer, "The Bay Area Research Wireless Access Network (BARWAN)" in Proceedings of the 4lst IEEE Computer Societv International Conference (COMPCON), 1996.

[19] S. Paviopoulos, E. Kyriacou, A. Berier, S. Dembeyiotis, and D. Koutsouris, "A novel emengency telemedicine system based on wireless communication technology-AMBULANCE," IEEE Transactions on Information Technologv in Biomedicine, vol. 2, no. 4, pp. 261-267, 1998.

[20] J. H. van Bemmel and M. A. Musen, Eds., Handbook of medical informatics. Heidelberg: Springer-Verlag.

[21] P. Szolovits, J. Doyle, J. William, I. S. Kohane, and S. G. Pauker, "Guardian angel: Patient-centered health information systems," MIT Laboratory for Computer Science, Tech. Rep. TR-604, May 1994.

[22] A. Naszlady and J. Naszlady, "Patient health record on a smart card," International Journal of Medical Informatics, vol. 48, no. 1-3, pp. 191194, 1998.

[23] P. Gunby, "Test of a new medical dog tag with civilian potential," Journal of the American Medical Association, vol. 279, no. 2, pp. 99 $100,1998$.

[24] T. Huston, "Security issues for implementation of e-medical records," Communications of the ACM, vol. 44, no. 9, pp. 89-94, 2001.

[25] I. Chlamtac and J. Redi, "Mobile computing: Challenges and potential," in Encyclopedia of Computer Science, 4th ed. International Thomson Publishing, 1997.

[26] C. D. Knutson, D. Joos, and R. Woodings, "The case for wireless independence: When access isn't the killer app." 2001.

[27] R. W. Woodings, "Quality of Transport (QOT): Dynamic transpor switching in WPANs," Master's thesis, Brigham Young University, 2002

[28] E. S. Hall, D. K. Vawdrey, and C. D. Knutson, "RF Rendez-Blue: Reducing power and inquiry costs in Bluetooth-enabled mobile systems," in Proceedings of the IIth IEEE International Conference on Computer Communications and Networks (ICCCN), Oct. 2002.

[29] T. C. Rindfieisch, "Privacy, information technology, and health care" Communications of the $A C M$, vol. 40, no. 8, pp. 92-100, 1997. 